\title{
Mycobacterial lymphadenitis without granuloma formation in a patient with anti-interferon-gamma antibodies
}

\author{
Mizuki Asako ${ }^{1} \cdot$ Hitomi Matsunaga ${ }^{1} \cdot$ Wataru Nakahara ${ }^{1} \cdot$ Mako Ikeda $^{1} \cdot$ Fuka Mima ${ }^{1} \cdot$ Ryota Minami $^{1}$. \\ Masahiro Sekiguchi ${ }^{2} \cdot \mathrm{Kazumasa} \mathrm{Oka}^{3} \cdot \mathrm{Naoki} \mathrm{Wada}^{4} \cdot \mathrm{Katsuhiro} \mathrm{Suzuki}^{5} \cdot \mathrm{Kazutaka} \mathrm{Yoshizawa}^{6} \cdot$ Takuro Sakagami $^{7}$. \\ Shuji Ueda ${ }^{1}$ (D)
}

Received: 30 March 2021 / Revised: 27 July 2021 / Accepted: 27 July 2021 / Published online: 30 July 2021

(c) Japanese Society of Hematology 2021

\begin{abstract}
A previously healthy 49 -year-old Japanese woman presented with cervical lymph node swelling and tenderness. Lymph node biopsy revealed reactive lymphadenitis without granulomas. No malignant cells were found, and no acid-fast positive bacilli were identified by Ziehl-Neelsen staining. She was treated unsuccessfully with various antibiotics, and it was very challenging to reach a diagnosis. ${ }^{18} \mathrm{~F}$-Fluorodeoxyglucose $\left({ }^{18} \mathrm{~F}-\mathrm{FDG}\right)$ uptake in bones was evaluated using positron emission tomography-computed tomography (PET-CT), and disseminated mycobacterial infection was suspected. The interferongamma (IFN- $\gamma$ ) release assays QuantiFERON (QFT) and T-SPOT were used to diagnose tuberculosis infection. On testing, a difference in mitogen response was found between these assays. The response was low for QFT but adequate for T-SPOT, suggesting the presence of anti-IFN- $\gamma$ antibodies. This difference depended on whether the patient's plasma (including antiIFN- $\gamma$ antibodies) was used within the assay system. Mycobacterium abscessus was isolated from lymph node cultures, and plasma anti-IFN- $\gamma$ antibodies were confirmed. The patient was diagnosed with disseminated M. abscessus infection with underlying adult-onset immunodeficiency caused by anti-IFN- $\gamma$ antibodies. Granulomas are a pathological hallmark of mycobacterial infection, but may not fully form in immunodeficient patients. Clinicians should be aware of the possibility of mycobacterial infection without granuloma formation due to anti-IFN- $\gamma$ antibodies.
\end{abstract}

Keywords IFN- $\gamma$ release assay $\cdot$ QuantiFERON $\cdot$ T-SPOT $\cdot$ Mitogen response $\cdot$ Anti-interferon-gamma antibodies · Mycobacterial infection

\section{Introduction}

Granuloma formation is a pathological hallmark of mycobacterial infection. Acid-fast bacillus staining methods, such as Ziehl-Neelsen staining, are useful for diagnosing mycobacterial lymphadenitis, but unfortunately have low detection rates $[1,2]$. In the case of mycobacterial lymphadenitis negative for acid-fast bacillus staining, the presence of granulomatous lesions on pathological examination becomes even more important. However, insufficient granuloma formation may be observed in an immunodeficient state [3].

Anti-interferon-gamma (IFN- $\gamma$ ) autoantibodies have been recognized as a cause of adult-onset immunodeficiency for mycobacterial infection, especially nontuberculous

Shuji Ueda

ueda0904@hp.pref.hyogo.jp

Extended author information available on the last page of the article mycobacterial infections $[4,5]$. Here, we report a case of disseminated Mycobacterium abscessus infection with underlying adult-onset immunodeficiency caused by anti-IFN- $\gamma$ antibodies. Mycobacterial lymphadenitis without granuloma formation was observed, which occurred due to the immunodeficiency associated with anti-IFN- $\gamma$ antibodies. The discrepancy in mitogen response between QuantiFERON (QFT) and T-SPOT assays aided the diagnosis of anti-IFN- $\gamma$ antibodies in this case.

\section{Case presentation}

A previously healthy 49-year-old Japanese woman presented with a 3-month history of swelling and tenderness of the left cervical lymph nodes. Associated symptoms included lowgrade fever and fatigue. Laboratory investigations revealed elevated inflammatory markers, including C-reactive protein 
$(4.27 \mathrm{mg} / \mathrm{dL})$ and serum soluble interleukin-2 receptor (1564 $\mathrm{U} / \mathrm{mL}$ ). Liver and renal function tests were unremarkable, except for albumin levels, which were lower than normal $(1.9 \mathrm{~g} / \mathrm{dL})$. Human immunodeficiency virus (HIV) antibody test was negative. As we initially suspected infectious inflammatory lymphadenitis, we began administering antibiotics, including garenoxacin, minocycline, and clarithromycin, but the patient's clinical symptoms worsened. Positron emission tomography-computed tomography (PET-CT) revealed pathological ${ }^{18} \mathrm{~F}$-fluorodeoxyglucose $\left({ }^{18} \mathrm{~F}-\mathrm{FDG}\right)$ uptake in the left cervical, left supraclavicular, bilateral axillary, left hilar, mediastinal, and left cardiac lymph nodes. It also detected bone lesions, mainly in the sternum (Fig. 1). Therefore, we performed cervical lymph node biopsy to exclude malignant lymphoma. The biopsy tissue was evaluated by pathological analysis of frozen sections, immunohistochemistry, flow cytometry, fluorescence in situ hybridization (FISH), and chromosomal analysis. Staining of sections with hematoxylin and eosin showed reactive lymphadenitis with abscess formation. No granulomatous lesions were present in the lymph nodes (Fig. 2) and Ziehl-Neelsen staining revealed no acid-fast bacilli. No evidence of malignant lymphoma was found on immunohistochemical staining, flow cytometry, FISH, or chromosomal analysis. Blood, urine, and sputum cultures were all negative for bacteria, fungi, and mycobacteria. Based on these initial results, we made a diagnosis of nonspecific inflammatory lymphadenitis. However, despite continued intravenous administration of antibiotics, including levofloxacin and cefepime, the lymphadenopathy and fever worsened.

As PET-CT showed ${ }^{18} \mathrm{~F}$-FDG uptake in the sternum (among other bones) rather than the lymph nodes, we attempted sternal bone marrow aspiration at the location of ${ }^{18} \mathrm{~F}$-FDG accumulation. However, despite several attempts, we failed to obtain bone marrow cells. Such an outcome is referred to as a "dry tap" and suggests a pathological process. The clinical findings were consistent with osteomyelitis, and we considered this to be the cause of ${ }^{18} \mathrm{~F}-\mathrm{FDG}$ uptake in the bone [6]. Osteomyelitis is usually caused by bacterial infection, although in rare cases it may be caused by fungal or mycobacterial infection in immunosuppressed patients [7-9]. Osteomyelitis is known to be an occasional complication of tuberculosis [10]. Recently, multifocal osteomyelitis after BCG vaccination has been reported in people with Mendelian susceptibility to mycobacterial diseases (MSMD), especially in cases of IFN- $\gamma \mathrm{R} 1$ deficiency $[11,12]$. To test for Mycobacterium tuberculosis (MTB) infection in our patient, we performed IFN- $\gamma$ release assays (IGRAs) with QFT-TB Gold Plus (QFT-Plus) and T-SPOT. The IFN- $\gamma$ level in the QFT-Plus
Fig. 1 a Positron emission tomography-computed tomography demonstrated ${ }^{18} \mathrm{~F}$-fluorodeoxyglucose uptake by multiple lymph nodes and bone lesions. b Cervical lymph node. c Axillary lymph node and bone lesion (in the sternum). d Hilar lymph nodes and bone lesion (in a vertebra)

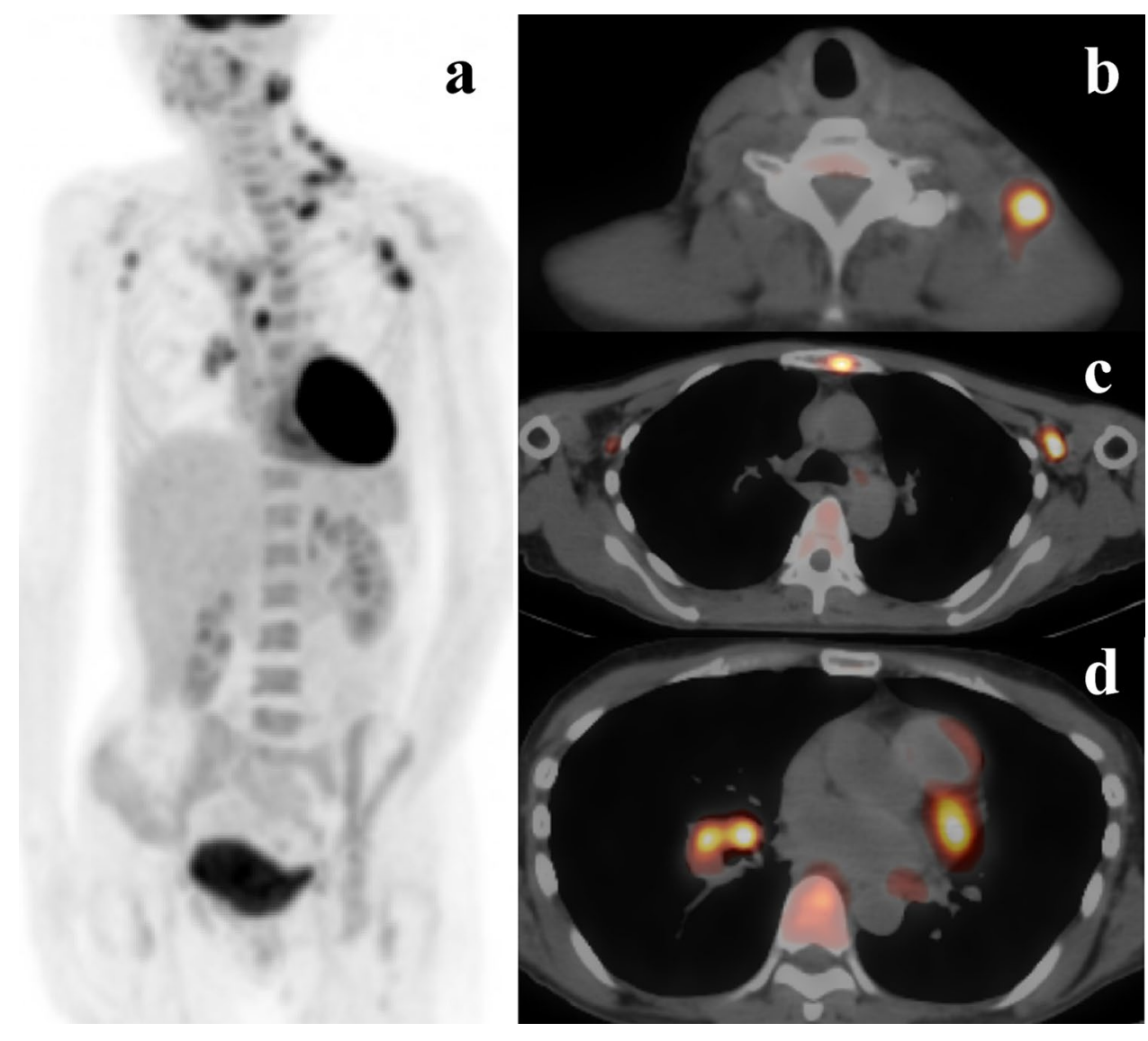



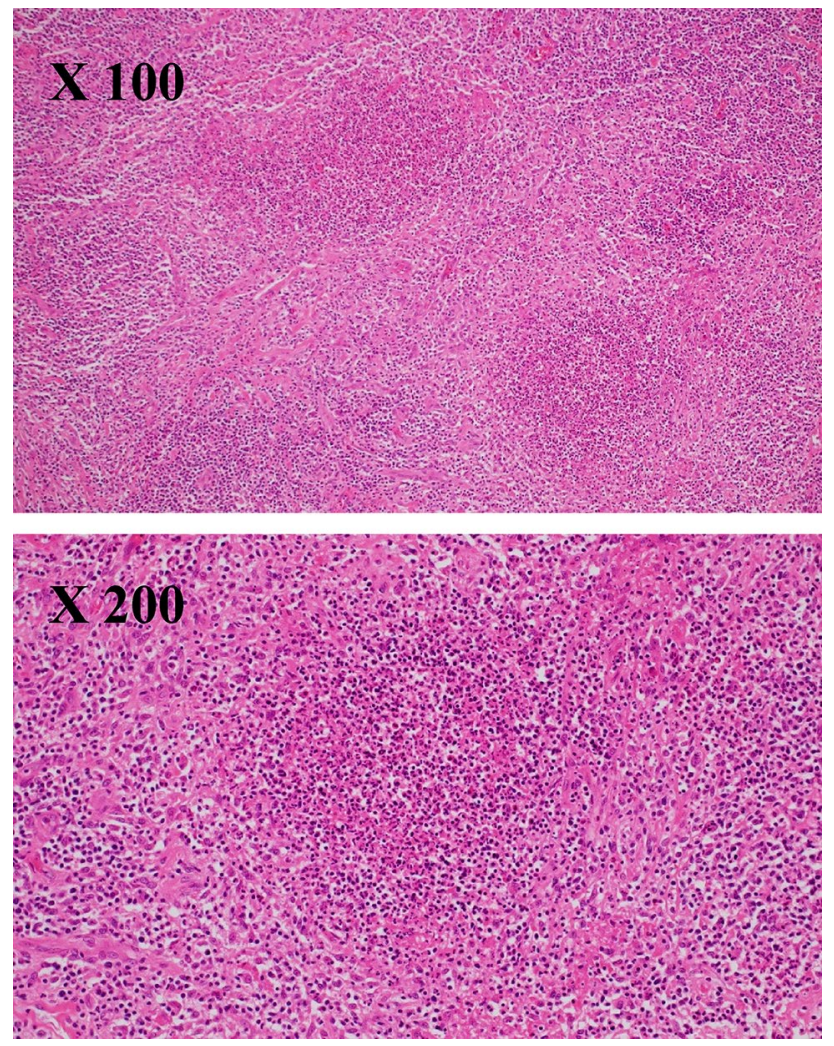

Fig. 2 Lymph node biopsy showed lymphadenitis with abscess formation. No granuloma was found in the lymph node (hematoxylin and eosin staining)

mitogen control was extremely low (enzyme-linked immunosorbent assay [ELISA] value $=0.09 \mathrm{IU} / \mathrm{ml})$, but high in the T-SPOT mitogen control ( $>20$ spots) (Table 1). This discrepancy strongly suggested that IFN- $\gamma$ was being inhibited by factors in the patient's plasma. Therefore, we performed a repeat cervical lymph node biopsy, followed by bacterial and mycobacterial culture test on the tissue. The histopathological finding was identical to the initial lymph node biopsy, and no granuloma formation was evident. Polymerase chain reaction tests of the lymph node for Mycobacterium tuberculosis, M. avium, and M. intracellulare were all negative, but Mycobacterium abscessus was isolated from the mycobacterial culture. Based on these findings, we made a diagnosis of disseminated nontuberculous mycobacterial infection (DNTM) caused by M. abscessus infection. The presence of anti-IFN- $\gamma$ antibodies was confirmed by analysis of IFN- $\gamma$-neutralizing antibody titer and IFN- $\gamma$-neutralizing activity at Niigata University Medical and Dental Hospital [13]. A high titer of neutralizing autoantibodies to IFN- $\gamma$ (concentration of anti-IFN- $\gamma$ : 141.5 E.U., control 22.9 E.U.; STAT1 phosphorylation index: -6.9 , control: 396) was identified in the patient's serum by ELISA and a flow cytometry-based method. The final diagnosis was disseminated M. abscessus infection with underlying adult-onset immunodeficiency caused by anti-IFN- $\gamma$ antibodies.

The patient was treated for the disseminated M. abscessus infection with combination chemotherapy consisting of meropenem (1000 mg/day), amikacin (600 mg/day), and clarithromycin $(800 \mathrm{mg} / \mathrm{day})$. One month after initiation of antimycobacterial treatment, the patient was afebrile and her lymphadenopathy had shrunk markedly. Treatment was continued on an outpatient basis with daily clarithromycin and an infusion of meropenem and amikacin three times a week. Three months later, PET-CT showed no abnormal ${ }^{18} \mathrm{~F}$-FDG accumulation. Antimicrobial treatment was continued for 1 year. At the time of writing, no regression was observed and the patient's condition remained stable for at least 6 months after the antimicrobial treatment was discontinued.

Table 1 The discrepancy in mitogen response between QuantiFERON-TB Gold Plus (QFT-Plus) and T-SPOT.TB (T-SPOT) in a female patient with disseminated Mycobacterium abscessus infection

\begin{tabular}{llc}
\hline QFT-Plus & Measured value (IU/mL) & $\begin{array}{l}\text { Normal standard } \\
\text { (IU/mL) }\end{array}$ \\
\hline Negative control & 0.02 & $\leq 8.0$ \\
TB1 stimulation & 0.00 & $<0.35$ \\
TB2 stimulation & 0.00 & $<0.35$ \\
Mitogen stimulation & 0.09 & $\geq 0.50$ \\
\hline T-SPOT & & Normal standard \\
& Spot number & $\leq 10$ \\
Negative control & 0 & $\leq 4$ \\
EST-6 stimulation & 0 & $\leq 4$ \\
CFP10 stimulation & 0 & $\geq 20$ \\
Mitogen stimulation & $\geq 20$ & \\
\hline
\end{tabular}

$T B 1$ long peptides of ESAT- 6 and CFP-10, TB2 TB1 and short peptides of CFP-10 


\section{Discussion}

DNTM mostly occurs as an opportunistic infection in immunosuppressed individuals, including those with advanced HIV infection [14]. Anti-IFN- $\gamma$ antibodies have recently been reported to affect the IFN- $\gamma$ immune pathway and cause adult-onset immunodeficiency syndrome in DNTM and other opportunistic infections, especially in East Asia $[4,5,15-17]$. The course of such infections in patients with this syndrome can be severe and progressive despite prolonged antimicrobial therapy, and treatment may fail [18, 19]. Anti-IFN- $\gamma$ antibodies are detected in about $80 \%$ of DNTM patients without obvious immunodeficiency, such as HIV infection $[4,5]$. Therefore, if a previously healthy person develops DNTM, the majority of cases are considered to have acquired immunodeficiency caused by antiIFN- $\gamma$ antibodies. The commonly involved organs include the lymph nodes (79\%), bone/joints lesions (34\%), and lungs (32\%) [20]. As DNTM in HIV infection rarely causes bone lesions, the presence of bone lesions may be suggestive of an association with anti-IFN- $\gamma$ antibodies [20-22]. For patients with both lymphadenopathy and bone involvement, DNTM-associated anti-IFN- $\gamma$ antibodies is one of the most important differential diagnoses. In our case, focusing on the presence of bone lesions provided insight that allowed us to reach the correct diagnosis.

Granulomas are a pathological hallmark of mycobacterial infection. Their main function is to localize and contain mycobacteria, while limiting the immune response to a relatively small area. Therefore, granulomas formation may be insufficient in an immunodeficient state [3]. As antiIFN- $\gamma$ antibodies cause an immunodeficient state specific to mycobacteria, it is possible that granuloma formation may be insufficient in this disease. In fact, a previous case report described mycobacterial infections in which granuloma formation was not observed [23]. However, this previous case report was published before anti-IFN- $\gamma$ antibodies became widely known, and this case may have been associated with anti-IFN- $\gamma$ antibodies. It is very difficult to diagnose mycobacterial infection without granuloma formation on pathological examination without performing mycobacterial culture. A number of DNTM cases with insufficient granuloma formation may have been overlooked.

If the presence of anti-IFN- $\gamma$ antibodies is confirmed, mycobacterial infection should be suspected even in the absence of granuloma formation. Recently, the QFT IGRA, which has been used extensively for in vitro diagnosis of MTB infection, has been repurposed for screening of patients with suspected anti-IFN- $\gamma$ antibodies [24, 25]. IGRAs are blood tests that detect the secretion of IFN- $\gamma$ ex vivo by assessing lymphocytes after stimulation with two or three proteins that are fairly specific for MTB. IGRAs using the M. tuberculosis-specific antigens ESAT-6 and CFP-10 have high specificity for the diagnosis of tuberculosis and rarely yield false positive for most nontuberculous mycobacterial infections, including M. abscessus [26, 27]. However a small number of nontuberculous mycobacterial infections, such as M.kansasii, M. marinum, and M. szulgai, which express ESAT-6 and CFP-10, can cause positive IGRA results [28]. Currently, two types of FDA-approved IGRA are available: the QFT assay (Qiagen) and T-SPOT assay (Oxford Immunotec). The QFT assay measures the concentration of IFN- $\gamma$ by ELISA, whereas T-SPOT measures the number of IFN- $\gamma$-secreting lymphocytes by enzymelinked immunospot (ELISPOT) assay [29, 30]. In addition to measuring IFN- $\gamma$ in the antigen-simulated sample, IGRAs also measure IFN- $\gamma$ levels in positive and negative controls. The negative control contains no additives and is used to adjust for background IFN- $\gamma$. In the positive control, mitogen, which is a strong, nonspecific T cell stimulant, is used to stimulate lymphocytes, thus controlling for IFN- $\gamma$ release that is unrelated to the tuberculosis antigen. A lack of IFN- $\gamma$ in the positive control usually implies that the patient's $\mathrm{T}$ cells do not respond to nonspecific IFN- $\gamma$-releasing antigens. As IGRAs assess IFN- $\gamma$ release, a common explanation for a negative result in the positive control is an immunocompromised state, for example due to corticosteroid treatment, HIV infection, or cancer [31]. The QFT assay quantifies the IFN- $\gamma$ released from sensitized lymphocytes in whole blood containing the patient's plasma incubated with MTB antigens. Therefore, in addition to an immunocompromised state, a lack of IFN- $\gamma$ in the positive control in the QFT assay may also indicate the presence of anti-IFN- $\gamma$ antibodies. This cannot be the case in the T-SPOT assay, as it counts the number of IFN- $\gamma$-secreting cells, which are peripheral blood mononuclear cells separated from whole blood by centrifugation, a process that removes the patient's plasma. Immunocompromised patients who do not have a positive control in the QFT assay are likely to not have a positive control in the T-SPOT assay. Therefore, it is recommended that the QFT and T-SPOT assays be used together to test for anti-IFN- $\gamma$ antibodies. T-SPOT assay alone does not indicate the presence of anti-IFN- $\gamma$ antibodies, and even if the QFT alone suggests the possibility of anti-IFN- $\gamma$ antibodies, it may be erroneously considered to be due to an immunodeficient state. Thus, a low mitogen response in the QFT assay together with an adequate mitogen response in the T-SPOT assay, as in the case presented here, strongly suggests the presence of anti-IFN- $\gamma$ antibodies. In our patient, we confirmed the diagnosis based on IFN- $\gamma$-neutralizing autoantibody titer and analysis of IFN- $\gamma$-neutralizing activity, which are specialized tests that can only be performed at specialist facilities [13]. The combination of QFT and T-SPOT allows the presence of anti-IFN- $\gamma$ antibodies to be confirmed using these widely available tests. 
In conclusion, granulomas are a pathological hallmark of mycobacterial infection, but may not fully form in an immunodeficient state. Clinicians should be aware of the possibility of mycobacterial infections without granuloma formation due to anti-IFN- $\gamma$ antibodies. Diagnosis of these cases is very challenging but the clinical findings of lymphadenitis with bone involvement, and the discrepancy in mitogen response between QFT and T-SPOT assays, are helpful.

\section{Declarations}

Conflict of interest All authors have no conflict of interest related to this manuscript.

\section{References}

1. Abdissa K, Tadesse M, Abdella K, Bekele A, Bezabih M, Abebe G. Diagnostic performance of fluorescent light-emitting diode microscopy for tuberculous lymphadenitis in a high-burden setting. Trop Med Int Health. 2015;20:1543-8.

2. Tadesse M, Abebe G, Abdissa K, Bekele A, Bezabih M, Apers $\mathrm{L}$, et al. Concentration of lymph node aspirate improves the sensitivity of acid fast smear microscopy for the diagnosis of tuberculous lymphadenitis in Jimma, southwest Ethiopia. PLoS ONE. 2014;9:e106726.

3. North RJ, Izzo AA. Granuloma formation in severe combined immunodeficient (SCID) mice in response to progressive BCG infection. Tendency not to form granulomas in the lung is associated with faster bacterial growth in this organ. Am J Pathol. 1993;142:1959-66.

4. Aoki A, Sakagami T, Yoshizawa K, Shima K, Toyama M, Tanabe $\mathrm{Y}$, et al. Clinical significance of interferon-gamma neutralizing autoantibodies against disseminated nontuberculous mycobacterial disease. Clin Infect Dis. 2018;66:1239-45.

5. Browne SK, Burbelo PD, Chetchotisakd P, Suputtamongkol Y, Kiertiburanakul S, Shaw PA, et al. Adult-onset immunodeficiency in Thailand and Taiwan. N Engl J Med. 2012;367:725-34.

6. Dioguardi P, Gaddam SR, Zhuang H, Torigian DA, Alavi A. FDG PET assessment of osteomyelitis: a review. PET Clin. 2012;7:161-79.

7. Gamaletsou MN, Rammaert B, Bueno MA, Moriyama B, Sipsas NV, Kontoyiannis DP, et al. Aspergillus osteomyelitis: epidemiology, clinical manifestations, management, and outcome. J Infect. 2014;68:478-93.

8. Petitjean G, Fluckiger U, Scharen S, Laifer G. Vertebral osteomyelitis caused by non-tuberculous mycobacteria. Clin Microbiol Infect. 2004;10:951-3.

9. Carek PJ, Dickerson LM, Sack JL. Diagnosis and management of osteomyelitis. Am Fam Physician. 2001;63:2413-20.

10. Vohra R, Kang HS, Dogra S, Saggar RR, Sharma R. Tuberculous osteomyelitis. J Bone Joint Surg Br. 1997;79:562-6.

11. Staines-Boone AT, Deswarte C, Venegas Montoya E, SanchezSanchez LM, Garcia Campos JA, Muniz-Ronquillo T, et al. Multifocal recurrent osteomyelitis and hemophagocytic lymphohistiocytosis in a boy with partial dominant IFN-gammaR1 deficiency: case report and review of the literature. Front Pediatr. 2017;5:75.

12. Bustamante J, Boisson-Dupuis S, Abel L, Casanova JL. Mendelian susceptibility to mycobacterial disease: genetic, immunological, and clinical features of inborn errors of IFN-gamma immunity. Semin Immunol. 2014;26:454-70.
13. Shima K, Sakagami T, Tanabe Y, Aoki N, Moro H, Koya T, et al. Novel assay to detect increased level of neutralizing antiinterferon gamma autoantibodies in non-tuberculous mycobacterial patients. J Infect Chemother. 2014;20:52-6.

14. Sharma SK, Upadhyay V. Epidemiology, diagnosis \& treatment of non-tuberculous mycobacterial diseases. Indian J Med Res. 2020;152:185-226.

15. van de Vosse E, van Dissel JT, Ottenhoff TH. Genetic deficiencies of innate immune signalling in human infectious disease. Lancet Infect Dis. 2009;9:688-98.

16. Filipe-Santos O, Bustamante J, Chapgier A, Vogt G, de Beaucoudrey L, Feinberg J, et al. Inborn errors of IL-12/23- and IFN-gamma-mediated immunity: molecular, cellular, and clinical features. Semin Immunol. 2006;18:347-61.

17. Kampmann B, Hemingway C, Stephens A, Davidson R, Goodsall A, Anderson S, et al. Acquired predisposition to mycobacterial disease due to autoantibodies to IFN-gamma. J Clin Invest. 2005; $115: 2480-8$.

18. Angkasekwinai N, Suputtamongkol Y, Phoompoung P, Pithukpakorn M, Wongswat E, Umrod P, et al. Clinical outcome and laboratory markers for predicting disease activity in patients with disseminated opportunistic infections associated with anti-interferon-gamma autoantibodies. PLoS ONE. 2019;14:e0215581.

19. Nishimura T, Fujita-Suzuki Y, Yonemaru M, Ohkusu K, Sakagami T, Carpenter SM, et al. Recurrence of disseminated Mycobacterium avium complex disease in a patient with antigamma interferon autoantibodies by reinfection. J Clin Microbiol. 2015;53:1436-8.

20. Hase I, Morimoto K, Sakagami T, Ishii Y, van Ingen J. Patient ethnicity and causative species determine the manifestations of anti-interferon-gamma autoantibody-associated nontuberculous mycobacterial disease: a review. Diagn Microbiol Infect Dis. 2017;88:308-15.

21. Kaneshiro K, Takatsuki K, Kanamori K. A healthy adult with disseminated nontuberculous mycobacterial infection with multiple bone lesions. Kekkaku. 2015;90:457-61.

22. Kalayjian RC, Toossi Z, Tomashefski JF Jr, Carey JT, Ross JA, Tomford JW, et al. Pulmonary disease due to infection by Mycobacterium avium complex in patients with AIDS. Clin Infect Dis. 1995;20:1186-94.

23. Kakugawa T, Mukae H, Kajiki S, Tanaka A, Yamayoshi T, Inoue $\mathrm{M}$, et al. Mycobacterium avium pleuritis in a non-immunocompromised patient. Intern Med. 2008;47:1727-31.

24. Wu UI, Chuang YC, Sheng WH, Sun HY, Jhong YT, Wang JY, et al. Use of QuantiFERON-TB Gold In-tube assay in screening for neutralizing anti-interferon-gamma autoantibodies in patients with disseminated nontuberculous mycobacterial infection. Clin Microbiol Infect. 2018;24:159-65.

25. Suarez I, Lehmann C, Gruell H, Graeb J, Kochanek M, Fatkenheuer G, et al. Repurposing QuantiFERON for detection of neutralizing interferon-gamma autoantibodies in patients with nontuberculous mycobacterial infections. Clin Infect Dis. 2017:65:518-21.

26. Takasaki J, Manabe T, Morino E, Muto Y, Hashimoto M, Iikura $\mathrm{M}$, et al. Sensitivity and specificity of QuantiFERON-TB Gold Plus compared with QuantiFERON-TB Gold In-Tube and T-SPOT.TB on active tuberculosis in Japan. J Infect Chemother. 2018;24:188-92.

27. Siegel SAR, Cavanaugh M, Ku JH, Kawamura LM, Winthrop KL. Specificity of QuantiFERON-TB plus, a new-generation interferon gamma release assay. J Clin Microbiol. 2018. https://doi.org/10. 1128/JCM.00629-18.

28. Andersen P, Munk ME, Pollock JM, Doherty TM. Specific immune-based diagnosis of tuberculosis. Lancet. 2000;356:1099-104. 
29. Mazurek GH, Jereb J, Vernon A, LoBue P, Goldberg S, Castro $\mathrm{K}$, et al. Updated guidelines for using interferon gamma release assays to detect mycobacterium tuberculosis infection-United States, 2010. MMWR Recomm Rep. 2010;59:1-25.

30. Arend SM, Thijsen SF, Leyten EM, Bouwman JJ, Franken WP, Koster BF, et al. Comparison of two interferon-gamma assays and tuberculin skin test for tracing tuberculosis contacts. Am J Respir Crit Care Med. 2007;175:618-27.

31. Ferrara G, Losi M, Meacci M, Meccugni B, Piro R, Roversi P, et al. Routine hospital use of a new commercial whole blood interferon-gamma assay for the diagnosis of tuberculosis infection. Am J Respir Crit Care Med. 2005;172:631-5.

Publisher's Note Springer Nature remains neutral with regard to jurisdictional claims in published maps and institutional affiliations.

\section{Authors and Affiliations}

\section{Mizuki Asako ${ }^{1}$. Hitomi Matsunaga ${ }^{1} \cdot$ Wataru Nakahara ${ }^{1} \cdot$ Mako Ikeda $^{1} \cdot$ Fuka Mima ${ }^{1} \cdot$ Ryota Minami $^{1}$. Masahiro Sekiguchi ${ }^{2} \cdot \mathrm{Kazumasa} \mathrm{Oka}^{3} \cdot \mathrm{Naoki} \mathrm{Wada}^{4} \cdot \mathrm{Katsuhiro} \mathrm{Suzuki}^{5} \cdot \mathrm{Kazutaka}$ Yoshizawa $^{6} \cdot$ Takuro Sakagami $^{7}$. Shuji Ueda ${ }^{1}$}

Mizuki Asako

m.asako.92922@gmail.com

Hitomi Matsunaga

h-matsunaga@hp.pref.hyogo.jp

Wataru Nakahara

nakaharawataru15.2@gmail.com

Mako Ikeda

tarikihongan@rb4.so-net.ne.jp

Fuka Mima

mimafuka.75@gmail.com

Ryota Minami

ryotadoctor0424@yahoo.co.jp

Masahiro Sekiguchi

sekiguchi1704@hp.pref.hyogo.jp

Kazumasa Oka

oka1004@hp.pref.hyogo.jp

Naoki Wada

wadan@med.u-ryukyu.ac.jp

Katsuhiro Suzuki

suzukikyra@yahoo.ne.jp
Kazutaka Yoshizawa

kazu_t_328@yahoo.co.jp

Takuro Sakagami

stakuro@kumamoto-u.ac.jp

1 Department of Hematology, Hyogo Prefectural Nishinomiya Hospital, 13-9, Rokutanji-cho, Nishinomiya, Hyogo 662-0918, Japan

2 Department of Rheumatology, Hyogo Prefectural Nishinomiya Hospital, Nishinomiya, Hyogo, Japan

3 Department of Pathology, Hyogo Prefectural Nishinomiya Hospital, Nishinomiya, Hyogo, Japan

4 Department of Pathology and Oncology, Graduate School of Medicine, University of the Ryukyus, Okinawa, Japan

5 Department of Internal Medicine, National Hospital Organization Kinki-Chuo Chest Medical Center, Sakai, Japan

6 Department of Respiratory Medicine and Infectious Disease, Niigata University Medical and Dental Hospital, Niigata, Japan

7 Department of Respiratory Medicine, Faculty of Life Science, Kumamoto University, Kumamoto, Japan 